the same field. We are indebted to Mr. R. Line for first bringing this disease to their notice.

School of Agriculture, W. A. R. Dillon Weston. Cambridge. July 13.

\section{Sharp Eyespot of Wheat caused by Corticium (Rhizoctonia) Solani}

EYESPOT of wheat caused by Cercosporella herpotrichoides is widespread in East Anglia, where it causes considerable loss in yield ${ }^{1}$. Typical symptoms of the disease are oval brown-bordered lesions on the straws near soil level. Similar, but less regular, lesions caused by another fungus have been recog. nized at Rothamsted as a different disease since 1935. This was described in Holland and figured by Oort ${ }^{2}$, who called it sharp eyespot but did not identify the causal organism. Sprague ${ }^{3,4}$ found a Rhizoctonia causing similar symptoms on wheat in Oregon. Blair ${ }^{5}$, working with Rhizoctonia Solani, found that some Canadian strains when inoculated into wheat caused a stem girdling injury the photographs of which resemble those of sharp eyespot. Neither worker identified the lesions caused by Rhizoctonia as the sharp eyespot described by Oort.

We have obtained wheat plants with sharp eyespot resembling those figured by Oort, Sprague and Blair from many localities in England and Wales, and have consistently isolated from them a fungus of the Rhizoctonia type. We have inoculated wheat seedlings with six isolates from four districts; all produced sharp eyespot lesions, from which we reisolated the fungus. The six isolates have now been identified by Dr. Bisby, Imperial Bureau of Mycology, as strains of Corticium (Rhizoctonia) Solani.

The symptoms of sharp eyespot are rather like those of true eyespot, and care must be taken to distinguish between them. Sharp eyespot lesions tend to be more superficial than those of eyespot and are sometimes confined to the outer sheaths, though they may penetrate the straw; they often run up the stem to a height of several inches. Sharp eyespot has asymmetric lesions with sharply defined borders surrounding pale areas on which develop spreading patches of mycelium. This is at first light-coloured but later darkens to brown or purple. True eyespot differs in having regular oval lesions, generally within two inches of soil level ; they have less sharply defined borders, with black spots of mycelium concentrated in the middle of the lesions.

Although sharp eyespot has been found in many wheat fields in England and Wales and one heavily infected crop has been reported, it is seldom present in more than about 1 per cent of the straws. It does not increase with the frequency of wheat or barley in the rotations as does eyespot, which may infect as many as 80-90 per cent of the straws where wheat and barley have been grown often. In the first wheat crop after grass, eyespot is generally absent or rare, but sharp eyespot is found just as commonly as on old arable land.

Mary D. GLynne.

WENDy M. RITChIE.

Ròthamsted Experimental Station, Harpenden, Herts. July 13.

${ }^{1}$ Glynne, Mary D., Ann. Appl. Biol., 29, 254 (1942).

2 Oort, A. J. P., Tidsch. over Plantenziekten., 42, 179 (1936).

s Sprague, R., Phytopath., 24, 946 (1934).

- Sprague, R., Phytopath., 27, 798 (1937).

'Blair, I. D., Canad. J. Res., C, 20, 174 (1942).

\section{Petal-Colour Inheritance in Lychnis}

Investigations of petal-colour inheritance in Lychnis species have resulted in considerable confusion owing to insufficiently precise identification of the forms studied.

Numerous authors, from Bartram ${ }^{1}$ (1699-1777) to Winge ${ }^{2}$, have found the production of petal-anthocyanin to be dominant in crosses between the closely related $L$. alba Mill. (L. vespertina Sibth.), with white petals, and $L$. dioica L. em Mill., with reddish-purple petals (Shull reunites both under the latter name). By crossings in reciprocal directions I have fully confirmed this. In a back-cross between the $F_{1}$ and $L$. alba, segregation took place in the ratio of one plant with coloured petals to one with white petals.

Only Shull ${ }^{3}$ differs from this belief, postulating that the production of colour depends upon the action of two dominant genes, each of which can be carried latent in white-petalled individuals. No other author has confirmed this and so far as I am aware Shull has not repeated it.

The existence in Nature of individuals resembling $L$. alba but possessing coloured petals ( $L$. alba var. colorata Lange) might be advanced as support for Shull's theory, but several authors have noticed that these individuals are absent from areas from which $L$. dioica is also absent. Post ${ }^{4}$ does not mention them from Palestine and Sinaii, where $L$. dioica does not occur. I have investigated many individuals of this kind (in several cases in swarms) and circumstantial evidence from the localities, together with positive evidence from pollen-sterility counts, measurement of certain vegetative and floral parts and comparison with hybrids (raised in the experimental plot from artificial crosses) as well as herbarium material has convinced me that they are either hybrids between $L$. alba and $L$. dioica or descendants therefrom.

In both $L$. alba and $L$. dioica the production of anthocyanin in the vegetative epidermis and the calyx (especially of staminate plants) is marked. Experiment has shown that it may be caused to develop even further by increased insolation, waterlogging of the soil, etc. Such increase is reflected in greater depth of petal coloration in $L$. dioica as the plants are transferred from shade to full exposure and vice-versa.

Although the petal-colour of the $F_{1}$ hybrid is less intense than that of $L$. dioica when the progeny is grown under the same conditions as the parent, variation of these may upset its manifestation. The matter is further complicated by the accompaniment of anthoxanthin, which certainly acts as a co-pigment to the anthocyanin, causing a blueing of the reddishpurple colour of $L$. dioica. It is present to a greater extent in $L$. alba than $L$. dioica, as might be expected on the basis of Lawrence and ScottMoncrieff's ${ }^{5}$ theory of the common precursor and balance of anthocyanin and anthoxanthin. These factors are probably partly responsible for the great range of colour shown by $F_{2}$ generations.

After frost or rain and as the season progresses, the shade of the coloured petals becomes increasingly blue. These facts probably dispose of Shull's factors for "reddish-purple" and "bluish-purple".

A variety of $L$. dioica is known (L.d. var. albiflora Tin.) with white petals. Examination of this form from several sources, combined with herbarium investigations at the Royal Botanic Gardens (Kew), the University of Cambridge, the British Museum 